\title{
Energías renovables y movimientos sociales en América Latina
}

\author{
Renewable energy sources and social movements \\ in Latin America
}

\author{
Emma Mendoza* \\ Vadim Pérez ${ }^{* *}$
}

\section{Resumen}

En el presente artículo se examinan, por una parte, el desarrollo intensivo en América Latina de tres industrias de energía renovable: la eólica (en Brasil y México), la hidroeléctrica (en Brasil) y la del cultivo de soya en gran escala (en Argentina y Brasil), y, por la otra -los movimientos sociales que surgen como respuesta a la expansión de estas industrias. Se propone la existencia de una relación causal entre el cambio del marco interpretativo (GOFFMAN, 1974) de estos movimientos sociales y el impacto de cada una de las industrias mencionadas en la sociedad y el medio ambiente, y se examina el papel del Estado en este proceso. A partir del análisis de estos tres casos se introduce el concepto de presión como factor determinante para la adopción y el cambio paulatino del marco interpretativo de un movimiento social, vistos aquí como

Facultad de Ciencias Políticas y Sociales, Universidad de Colima, México <emma_mendoza@ucol.mx>.

** Instituto de Biotecnología, UNAM vadperez@ibt.unam.mex

Recibido el 5 de mayo de 2009; aceptado el 15 de enero de 2010. 
indicadores de los cambios evolutivos que tienen lugar en la sociedad latinoamericana contemporánea.

Palabras clave: América Latina, energías renovables, movimientos sociales, relación Estado-sociedad.

\section{Abstract}

This article examines, on one hand, the intensive development of three renewable energy industries in Latin America: wind (in Brazil and Mexico), hydropower (in Brazil) and large scale soy growing (in Argentina and Brazil) and, on the other, social movements that emerge in response to the expansion of these industries. The text posits the existence of a causal relation between frame changes (GOFFMAN, 1974) of these social movements and the impact of each of these industries on the environment and society. On the basis of the analysis of the three cases, the concept of pressure is introduced, as a key factor that determines the adoption and change of frame of a social movement, seen here as indicators of the evolutional changes in contemporary Latin American society.

KeYwords: Latin America, renewable energy, social movements, statesociety interrelations. 
Emma Mendoza eT aL. • Energías renovables y movimientos sociales...

\section{INTRODUCCIÓN}

Entendemos por «energía renovable» o «energía alternativa» aquellas fuentes de energía que se obtienen de fuentes naturales capaces de renovarse $y$ susceptibles de utilizarse indefinidamente cuando se administran de manera sustentable. Estas fuentes incluyen la energía solar, la eólica y la geotérmica, así como la energía proveniente de los desechos de la agricultura, de la leña, del estiércol, de los monocultivos y de distintos residuos orgánicos, utilizados ya sea directamente o en forma procesada. Entre las fuentes de energía renovables se distingue también entre las tradicionales, como la leña, el bagazo de caña, el estiércol, que se utilizan directamente como combustible para calentar agua o preparar alimentos y aquellas como la energía solar, eólica, geotérmica y la proveniente de la biomasa elaborada, cuyo aprovechamiento requiere un nivel tecnológico relativamente avanzado y que se conocen como energías renovables nuevas.

Entre las ventajas de las fuentes de energía renovables, en comparación con las fuentes energéticas tradicionales ${ }^{1}$, cabe mencionar: 1) su enorme potencial -cerca de 6 veces el consumo mundial actual de energía (Nitsch y cols., 2004); 2) el hecho de que son de producción nacional, es decir, no se requiere importarlas; 3) se producen en forma descentralizada (la energía es consumida en el lugar en que se

1 El petróleo, el gas natural y el carbón. produce), lo que confiere al sistema estabilidad y seguridad contra fallos; 4) la limpieza del producto obtenido, ya que la explotación de los recursos primarios de estas fuentes no produce contaminación significativa; y 5) los nuevos empleos en la industria high tech.

Por otra parte, estas fuentes tienen algunos inconvenientes que han determinado su baja participación en el suministro total de energía. Estos inconvenientes son: 1) los elevados costos de la inversión inicial necesaria y el alto riesgo que implican las inversiones ya que, por una parte, la cantidad de energía que se generará no está asegurada por la naturaleza y, por la otra, de acuerdo con el esquema actual de explotación, las empresas deben asegurar con antelación el precio de venta de la energía; 2) la potencia de salida de estos sistemas en pequeña escala es variable, pues la cantidad de energía generada depende de las condiciones ambientales locales, que son variables y 3) cuando las fuentes se explotan de manera no sustentable (como es el caso de las grandes industrias contemporáneas) provocan enormes daños a la sociedad y al medio ambiente, generando costos externalizados que por lo general no son considerados en el precio final de la energía generada.

A pesar de sus desventajas, se considera que las energías renovables son uno de los pilares del desarrollo energético en el mundo. Ello obedece a que la energía limpia que se genera a partir de estas fuentes puede contribuir a 
mitigar los efectos antropogénicos del sistema actual, que se basa primordialmente en la obtención de energía a partir de la combustión de fuentes fósiles, que producen grandes cantidades de contaminantes.

Gracias a sus condiciones geográficas y climatológicas, América Latina es una de las regiones que poseen alto potencial de generación a partir de fuentes de energía renovables. No obstante, de todas ellas, las fuentes tradicionales -la leña, el bagazo de caña, etc.- son las que tienen mayor participación en el suministro total de energía primaria, lo que pone de manifiesto el atraso tecnológico en el aprovechamiento de las fuentes nuevas de energía renovables que, por lo demás, son las que crecen con mayor fuerza en la región.

Otra característica del aprovechamiento de las fuentes de energía alternativas en América Latina es que actualmente su incremento corresponde más que nada a la expansión de grandes compañías centralizadas, estatales o mayoritariamente particulares, contando estas últimas con el sólido respaldo por parte de los gobiernos nacionales. El apoyo gubernamental otorgado a este tipo de empresas es una de las estrategias que se aplica en todo el mundo para fomentar el mercado de las energías renovables y, según la Convención Marco de las Naciones Unidas para el Cambio Climático (CMNUCC), persigue reducir el impacto de las actividades del hombre en el clima global.
La forma centralizada de explotar estas fuentes de energía renovables provoca la consiguiente disminución de los beneficios de su aprovechamiento descentralizado y la reducción de su impacto positivo en el desarrollo social en los planos local y nacional. Es más, este modelo de desarrollo incluso llega a afectar directamente a comunidades a través del desplazamiento forzado de habitantes del territorio en que se planea establecer el proyecto e indirectamente, mermando la posibilidad de satisfacer las necesidades básicas para la supervivencia y, en definitiva, obligando a los afectados a emigrar de sus lugares de residencia. Sin embargo, como consecuencia del modelo de desarrollo establecido, que involucra la inserción en gran escala de cambios en los mecanismos que proporcionan la sustentabilidad social y ambiental, en el último tiempo resultan afectadas la autosustentabilidad y las seguridades elementales de la población de países enteros ${ }^{2}$.

\footnotetext{
La naturaleza proporciona de manera natural, valga la redundancia, la sustentabilidad del medio ambiente. La sustentabilidad social local se considera lograda cuando el impacto de la comunidad humana en la sustentabilidad del medio ambiente local no produce alteraciones significativas (que interfieran con los mecanismos de renovación) en ella. Dentro de la autosustentabilidad de un país se consideran las condiciones dadas naturalmente que le permiten satisfacer por sus propios recursos las necesidades de supervivencia mínimas de su población (la presencia de agua, alimentos, lugares aptos para la vida).
} 
Emma Mendoza eT aL. • Energías renovables y movimientos sociales...

Por lo tanto, resulta hasta cierto punto paradójico que el avance de las grandes industrias de energía renovable provoque la oposición de quienes serían beneficiados por estos proyectos. Esta oposición se manifiesta contra formas inadecuadas de explotación de estas energías renovables, que no reditúan beneficios a las localidades en que se instalan estas industrias e incluso las afectan de manera directa.

Las tres industrias de energía renovable cuyo avance en la región de América Latina ha provocado movimientos sociales en su contra y que serán objeto de atención en el presente artículo son la eólica, la hidroeléctrica y la de los monocultivos de gran extensión (en el caso de la soya). Las tres son las que mayor crecimiento han experimentado en la región en los últimos años. No obstante, la presión que ejerce cada una de ellas en la sociedad y el medio ambiente es diferente, lo que a nuestro juicio ha sido una de las condiciones clave del tipo de respuesta de los movimientos sociales y sus demandas. Consideramos además que, en nuestro caso, el aumento de la magnitud de las presiones que ejercen las industrias de energía renovable y el respaldo y la legitimación que aportan a este proceso las decisiones del gobierno, provocan un cambio cualitativo en las demandas de los movimientos sociales de oposición, en la percepción popular acerca de los orígenes y la legitimidad de dichas presiones (cambio del marco interpretativo), y en la expansión del enfoque de las deman- das de los opositores. Las presiones mencionadas van desde la apropiación por el gobierno de las tierras de las pequeñas comunidades autóctonas que, siendo legal desde el punto de vista del concepto de la razón de Estado, provoca un cambio drástico en el modo de vida de las comunidades desplazadas, destruye valores como sitios sagrados y erradica actividades productivas sustentables orientadas al autoconsumo. Por otra parte, las presiones sobre la sociedad y el medio ambiente llegan incluso a subvertir las bases de la autosustentabilidad de países enteros, debilitando su seguridad alimentaria y emergieron como consecuencia de las reformas neoliberales que se promovieron en América Latina en la década de los años noventa. Como consecuencia de ello, varían las demandas de los movimientos sociales surgidos contra estas presiones de carácter nacional y, de una reclamación de justicia y una oposición a las industrias mencionadas pasan a un nivel superior en que empiezan a incluir elementos de rechazo al sistema en general, a la lógica neoliberal basada en el mercado que, a escala global, articula el desarrollo económico a expensas de la sociedad y el medio ambiente.

El presente artículo tiene como objetivo presentar de manera descriptiva las respuestas a las siguientes interrogantes: ¿Cuál es el impacto sobre la sociedad y el medio ambiente latinoamericanos de las tres mayores industrias de energía renovable -la eólica, la hidroeléctrica y la de los monocultivos? 
¿Cómo cambian las respuestas de los movimientos sociales frente a las presiones originadas por la expansión de estas industrias; existe alguna diferencia entre las respuestas originadas por las diferentes presiones socioambientales que implica el desarrollo de dichas industrias? Finalmente, ¿cuál es el papel que juega el Estado en este proceso y de qué manera su actitud moldea los movimientos sociales y sus demandas originadas por la actual forma de explotación de las energías renovables?

Para establecer la relación entre las presiones ejercidas por las industrias de energía renovable y la respuesta contraria a ellas de los movimientos sociales, se analiza el marco interpretativo de estos, elegido como reflejo de la realidad a la que se oponen los movimientos sociales, "una perspectiva desde la que una situación amorfa, mal definida y problemática puede quedar consignada con un sentido", siendo este proceso de interpretación de la realidad la base para poder «emprender una acción» (Rein y Schön, 1993:p.146).

El concepto de marco interpretativo (frame) fue introducido en la obra clásica de Erving Goffman «Frame analysis: an essay of the organization of experience» (1974) como el material y esquemas utilizados para interpretar, identificar, percibir y ubicar diferentes sucesos de la vida real que ayudan al sujeto a entender la realidad. Snow y Benford (1988 y trabajos posteriores) enfatizan la importancia de este concepto para la selección de la estrategia de los movimientos sociales, con el fin de lograr un mayor apoyo y una mayor resonancia en la sociedad. Según estos autores, la línea principal de los discursos, los símbolos, las acciones seguidas por los movimientos sociales son elaborados y elegidos conscientemente para ofrecer a la sociedad una nueva explicación de los acontecimientos vividos, esclareciendo sus causas y ofreciendo una propuesta de acción. No obstante, las aproximaciones teóricas a partir de las cuales Snow y Benford estudian los movimientos sociales no les permiten explicar por qué, en igualdad de condiciones, en algunos casos los sujetos se incorporan al movimiento social y en otros no. En el presente trabajo se incorpora el concepto de presiones para intentar explicar el surgimiento de los movimientos sociales de oposición a las industrias de energía renovable y el cambio que ellas originan en los marcos interpretativos de estos movimientos, en sus demandas y en las acciones que emprenden.

LOS TRES PILARES DEL

DESARROLLO DE LAS ENERGÍAS RENOVABLES EN AMÉRICA

LATINA: LAS INDUSTRIAS

EÓlICA, HIDROELÉCTRICA Y DE MONOCULTIVOS

\section{La industria eólica}

La industria eólica es la más joven y de menor alcance de las tres que se 
examinan en el presente estudio y por lo tanto, la que menor impacto ha ocasionado en el medio ambiente y la sociedad. Los dos países que actualmente cuentan con mayor capacidad de generación a partir de esta fuente de energía en la América Latina son Brasil (con $256 \mathrm{MW}$ ) y México (con $88 \mathrm{MW}$ ) (Lawea, 2008). En estos países, esta clase de energía se capta principalmente en parques eólicos construidos en los sitios que tienen el potencial apropiado.

El mayor parque eólico de América Latina se encuentra en Brasil -en el municipio de Osório-e incluye tres proyectos - «Sangradouro», "Osório» $\mathrm{y}$ «Dos Indios», que suman un potencial de generación de $150 \mathrm{MW}$. Por su parte, en México el más importante es el parque eólico "La Venta II», situado en el Istmo de Tehuantepec, en Oaxaca, cuyo potencial se aproxima a los $84 \mathrm{MW}$. Ambos proyectos son operados principalmente por capital extranjero de origen español: en Brasil, la principal empresa protagonista es Enerfin/Enervento, con un $90 \%$ de participación, mientras que en México el parque es concesionado a las empresas Gamesa Eólica e Iberdrola Ingeniería. A pesar de que sus condiciones son similares, el parque de Osório fue instalado tras cuatro años de exhaustivo estudio del impacto ambiental (Prefeitura, 2008) y no se comprobó que hubiese oposición abierta o demandas contra los proyectos, mientras que en México la puesta en marcha del parque eólico de «La Venta II» se ha acompañado de la oposición rotunda por parte de actores locales. Esta oposición surgió en 2003 cuando se construyó el parque eólico y ha sido provocada por varios factores de carácter económico, social y legal.

«La Venta II» es la segunda etapa de desarrollo del proyecto inicial de la Comisión Federal de Electricidad (CFE) «La Venta I» y es parte del corredor eoloeléctrico que se planea instalar en el Istmo de Tehuantepec, con una capacidad total de $2.000 \mathrm{MW}$ (Moreno, 2004). Para la construcción de esta obra se eligieron los terrenos que se encuentran en comunidades de Oaxaca como Juchitán, San Dionisio del Mar, Unión Hidalgo, Santo Domingo Ingenio, San Mateo del Mar, la mayor parte de cuya población es indígena y vive en condiciones de pobreza extrema (PNUD, 2007). La razón de la elección de estas localidades para la construcción de proyectos eólicos es el potencial excepcional de generación con que cuentan, que supera incluso el de sitios calificados de excelentes a nivel mundial. Este factor, al igual que las facilidades que ofrece el gobierno mexicano para las realización de inversiones extranjeras (básicamente la exención de impuestos) ha atraído a varias compañías, principalmente de origen español, a invertir en proyectos eólicos en Oaxaca. No obstante, la actitud del Estado mexicano ante esta posibilidad de impulsar el desarrollo económico de la región oaxaqueña ha sido decepcionante y ha sido el detonante principal del surgimiento de movimientos sociales que han llegado 
a exigir que se deje sin efecto cualquier proyecto eólico en el sitio.

¿Cuál es el origen de este naciente movimiento social contra una industria que a escala mundial es reconocida como una de las que ofrece mayores beneficios para generar energía limpia? Como ya se mencionó, el principal actor responsable del surgimiento del movimiento social opositor es el Estado mexicano, que ha desatendido el proceso de negociación entre las empresas inversoras y los habitantes del Istmo de Tehuantepec. Esta situación ha dado lugar a que las empresas abusen de pobladores que viven en condiciones de pobreza extrema y les ofrezcan precios que fluctúan entre 100 y 150 pesos por hectárea al año durante el proceso de construcción, precio que después se reemplazará por un pago de 4 mil pesos al año por torre instalada más el $1.5 \%$ de los ingresos de la electricidad generada (Castañeda y Van Der Fleit, 2007). En tanto, en el proyecto inicial «La Venta I», los pagos acordados fueron de mil pesos al año durante el proceso de construcción, más 8 mil pesos por torre instalada. Otro conflicto relacionado con las tierras deriva de su pertenencia a la comunidad, es decir, los lotes de tierra que pueden alquilarse a las compañías extranjeras no tienen un propietario único. El gobierno mexicano intentó resolver este problema mediante el programa nacional PROCEDE, otorgando certificados de derechos ejidales ${ }^{3}$, que a juicio de los movimientos sociales oaxaqueños constituyen una forma de privatización de la tierra comunal, por lo cual el programa fue rechazado por los opositores. Otro factor que ha provocado el conflicto entre los movimientos sociales de Oaxaca y las autoridades ha sido que el Gobierno no ha prestado ayuda ni proporcionado información a los habitantes que desean alquilar sus tierras para la construcción del parque eólico.

Los principales actores que se oponen de manera rotunda al corredor eólico son la Unión de Comunidades Indígenas de la Zona Norte del Istmo (UCIZONI) y los Centros de Derechos Humanos Tepeyec y Gubiña XXI, entre otros (Castañeda y Van Der Fleit, 2007). Las demandas formuladas durante el Foro Regional Parque Eólico del Istmo, en el año 2005, se centran en los siguientes puntos (PRONUNCIAMIENTO, 2005): la construcción y operación del corredor eólico por empresas privadas extranjeras será un acto de privatización y una violación a la Constitución, que establece como derecho perteneciente al pueblo mexicano la capacidad de generar y transmitir la energía generada; se exige suspender la ejecución de las obras hasta que se realice una verdadera consulta a los pueblos vinculados; se exige el respeto a la cultura y los derechos de los pueblos indígenas, anteponiéndo-

Programa de Certificación de Derechos Ejidales, puesto en marcha en 1993 con el fin de dar certidumbre jurídica a la tenencia de la tierra, regularizar los derechos

agrarios y otorgar certificados de propiedad individual a los ejidatarios (de Ita, 2003). 
los a cualquier proyecto, y se exige el acceso a información fidedigna sobre el contexto, impacto y riesgos de la instalación del corredor. Entre las irregularidades que señalan estos actores cabe mencionar la ausencia de estudios serios de impacto ambiental que incluyan una estimación de los efectos en las aves migratorias que pasan por el sitio y la ausencia de una consulta a los habitantes de las comunidades antes de construir el parque. La naturaleza de estas demandas revela un hecho significativo: mientras que en Brasil las autoridades prestaron atención a las cuestiones ambientales y sociales durante la construcción del proyecto eólico de Osório para evitar la oposición a este, en condiciones similares en México el gobierno se deslindó de sus responsabilidades sociales y traspasó a las empresas españolas el derecho a resolver los conflictos de intereses originados por la construcción del parque eólico. Analizando las demandas de los movimientos en contra del corredor se comprueba que aunque incluso se exige cancelar las obras, en las demás partes del pronunciamiento estas demandas se vinculan antes que nada con la exigencia de respeto por los derechos humanos de los habitantes de las comunidades y como comunidades indígenas. Las autoridades mexicanas desconocen las condiciones impuestas por las empresas para alquilar la tierra de las comunidades indígenas y en la mayoría de los casos no intervienen en el diálogo entre ambos actores. Incluso ocurre lo contrario: ante la resistencia de los ejidatarios a alquilar sus terrenos (hasta 2007 solo se había logrado obtener en arrendamiento un $40 \%$ de la superficie total requerida para construir el corredor eólico), la Comisión Federal de Electricidad inició una acción penal contra los ejidatarios de La Venta «por el presunto delito de impedimento en la ejecución de obra pública» (Castro, 2007). Hasta el momento, este conflicto de intereses no se ha resuelto, aunque permanece suspenso por retrasos en la construcción de líneas de transmisión, a cargo de la CFE.

\section{La industria hidroeléctrica}

La hidroelectricidad es la principal fuente de generación de electricidad en América Latina y en 2006 aportó cerca del $60 \%$ del total de la electricidad generada (IEE, 2007). Los bajos costos de operación y la alta eficacia de las centrales hidroeléctricas, así como el gran potencial con que cuenta la región han sido unos de los factores que han propiciado su amplia expansión por toda la región -actualmente solo Barbados, Granada y Trinidad y Tobago no cuentan con centrales hidroeléctricas. Brasil es el principal país productor, ya que aporta más del $35 \%$ del total de generación de la región, y un $83 \%$ de la energía que consume corresponde a hidroelectricidad (IEE, 2007). La expansión de las centrales hidroeléctricas en Brasil comienza en la década de 1970, época en que se construyeron grandes represas para alimentar indus- 
trias de uso intensivo de electricidad y estimular el crecimiento económico del país. Estas construcciones provocaron la pérdida de terrenos y empleos, así como el desalojo de millares de personas de sus tierras, que se vieron obligadas a trasladar su residencia a la periferia de las grandes ciudades, en búsqueda de posibilidades de vida (MAB, sitio web). El aumento de las oportunidades políticas que se da durante la transición del militarismo a la democracia entre 1979 y 1983 permitió que los afectados se organizaran como un movimiento social (Rothman, 1993). Siguen construyéndose represas, por lo cual la presión que ejercen en determinados sectores de la sociedad no solo no disminuye sino que incluso se extiende, ocasionando el aumento del número de afectados. Por su cercanía geográfica y unidos bajo una presión común, estos comienzan a relacionarse, a colaborar entre sí y a intercambiar información para oponer resistencia al modelo impuesto por el gobierno. Los tres focos principales de resistencia en el país, que iniciaron lo que años más tarde sería el Movimiento de los Afectados por las Represas (Movimento dos Atingidos por Barragens, MAB por su sigla en portugués), uno de los movimientos sociales más importantes de toda América Latina por su magnitud, alcance y capacidad de influir en el proceso de toma de decisiones. Los tres frentes de resistencia incluyeron: en la región noreste -la lucha y movilización popular contra la construcción de la central hidroeléctri- ca de Sobradinho, en el río São Francisco, que provocó el desalojo de más de 70 mil personas a fines de los años setenta; en la región sur -la oposición a la construcción de la represa de Itaipú sobre el río Paraná y a los planes de construir las represas de Machadinho e Ita sobre el río Uruguay, en 1978; y en la región norte -la organización del pueblo contra la represa Tucuruí, en el mismo período (MAB, sitio web). En el sur, las alianzas con la Iglesia católica progresista, un colegio local y asociaciones rurales iniciaron el movimiento institucionalizado como Comisión Regional de los Afectados por las Represas (Comissão Regional de Atingidos por Barragens, CRAB), pequeña organización creada para luchar contra un número reducido de grandes represas construidas en la región (McCormick, 2004). En 1957 la CRAB pudo influir en las políticas de Eletrosul (la empresa estatal de electricidad que opera en el sur de Brasil) y obligarla a pagar indemnizaciones o a reasentar a las poblaciones desplazadas, nombrando observadores para vigilar su cumplimiento.

Tras comenzar en 1979 como movimiento en pro de los derechos a la tierra ocupada por el área inundada de las represas, en la década siguiente el movimiento antirepresas de Brasil fue objeto de cambios paulatinos, mediante la incorporación primero, aparte de los directamente afectados por la inundación de sus tierras, a las personas que serían afectadas por planes de construcción de represas y lue- 
go -a los afectados indirectamente por ellas (Rothman y Oliver, 1999). Otro acontecimiento importante se produjo a fines de los años ochenta, cuando el movimiento entró en contacto con organizaciones del movimiento ambientalista internacional, lo que condujo a un cambio de su marco interpretativo y de un movimiento de reclamo sobre la tierra pasó a ser más amplio y fusionó las demandas sobre la tierra con demandas ambientales. En palabras de los activistas del propio movimiento, se dieron cuenta de que "más allá de la lucha por los derechos, deben luchar por un modelo energético más justo» (acerca de MAB, REDLAR, sitio web). A partir de ese momento los objetivos del movimiento son: 1) aumentar la participación democrática en la legislación energética, 2) cambiar la política energética y 3 ) modificar la percepción ciudadana acerca de las represas hidroeléctricas y sus alternativas, para lo cual estableció un nuevo ámbito de acción comunicacional (HABERMAS, 1986). La misión del movimiento consiste ahora en luchar contra la construcción de represas, proteger los derechos de los habitantes que viven en las proximidades de los ríos, incluyendo a los indígenas y los quilombos ${ }^{4}$, incrementar la conciencia ciudadana sobre los impactos sociales y ambientales de las grandes represas y proponer de alternativas para la generación de energía (McCormick, 2004).

4 Descendientes de los esclavos africanos fugitivos.
Los movimientos sociales comienzan a ver las represas no solamente como construcciones gigantescas que provocan daños sociales directos como desplazamientos, cambio de tradiciones y eliminación de sitios sagrados de las comunidades desplazadas, erradicación de las actividades de subsistencia en algunos sectores de la sociedad que los obligan a buscar otras alternativas para la supervivencia. También se considera su enorme impacto ambiental -las grandes cantidades de gases de efecto invernadero emitidas durante la descomposición de la materia orgánica inundada, cambios del sistema hidrográfico e impactos indirectos relacionados con los patrones actuales de uso de la energía, poniendo de relieve la necesidad no de incrementar la producción de energía ante la creciente demanda sino de fomentar su uso eficiente.

El movimiento antirepresas ha tenido tal trascendencia en América Latina (principalmente, debido al gran número de estas construcciones en la región), que en años recientes ha fortalecido los vínculos entre actores de diversos países de la región, formando redes de movimientos, como por ejemplo la Red Latinoamericana contra Represas y por los Ríos, sus Comunidades y el Agua (REDLAR), organización trasnacional que unifica los esfuerzos de actores individuales y respalda los movimientos sociales nacionales. Esta red, aparte de perseguir los objetivos ya mencionados, promueve también la lucha contra la privatización de los 
recursos acuíferos de los países latinoamericanos, práctica que se inició con la apertura de las economías de las naciones de la región a la inversión extranjera.

Otra característica del movimiento antirepresas en años recientes es su paulatina vinculación con otros movimientos sociales que comparten el mismo marco interpretativo -de afectados por el modelo económico basado en la apertura a las inversiones extranjeras y denominado por los movimientos sociales neocolonialismo debido a que se vincula con la exportación desmesurada de los recursos naturales de las naciones latinoamericanas. Ejemplo de este vínculo entre movimientos sociales se da en México, donde el Movimiento Mexicano de Afectados por las Presas y en Defensa de los Ríos (MAPDER) incluye en sus declaraciones la solidaridad con las demandas del movimiento contra la construcción del corredor eólico del Istmo de Tehuantepec (MAPDER, 2008).

\section{La industria de los monocultivos}

La tercera industria estudiada en el presente artículo es la de los grandes monocultivos que hoy en día se posicionan como una alternativa para la producción de agrocombustibles (Honty y Gudynas, 2007), sustitutos de los combustibles líquidos tradicionales para el transporte automotriz. En la actualidad existen dos tipos de agrocombustibles -el bioetanol y el biodiesel, derivados principalmente de la caña de azúcar y de la soya, respectivamente. Las exportaciones de bioetanol han permitido que Brasil se establezca como líder mundial en su producción y genera muchas expectativas sobre las posibilidades de crecimiento económico que ofrece esta industria para los países que deseen instalarla. No obstante, la industria del biodiesel o más específicamente el cultivo de soya que la sostiene es la que mayor auge está teniendo hoy en día y la que mayores presiones ejerce en el ambiente y la sociedad de los países del sur de la región, generando a su alrededor un ambiente de polémica respecto de su viabilidad como alternativa sustentable para combatir los problemas del cambio climático. Para formarnos una idea del impacto del cultivo de la soya en los países del Cono Sur, veamos los siguientes datos ${ }^{5}$.

Argentina y Brasil son los dos principales productores y exportadores de soya de la región, cultivo que ocupa cerca del $45 \%$ del área cultivada en Brasil (REPORTER BRASIL, 2008) y una cifra similar en Argentina (RULLI, 2007), es decir alrededor de la mitad de los terrenos cultivables de estos países se destina al cultivo de la soya para exportación, proporción que va en

\footnotetext{
Para una revisión más exhaustiva de las consecuencias de la producción de la soya en los países del sur de América Latina, ver, por ejemplo, los sitios web http:// www.agrcombustibles.org, del Centro Latinoamericano de Ecología Social, Uruguay y http://www.lasojamata.org, y http://www.grr.org.ar del Grupo de Reflexión Rural, Argentina.
} 
aumento. En Brasil, en la zafra 20072008, el área cultivada alcanzó los 21 millones de hectáreas (REPORTER BRASIL, 2008), mientras que en Argentina la cifra fue de 16 millones de hectáreas en el ciclo 2006-2007 (Rulli, 2007). Este hecho tiene varias implicaciones. Primero, la enorme competencia que hace la soya a los cultivos destinados al consumo humano, reduciendo la disponibilidad y aumentando los precios no solo de verduras y hortalizas, sino también de carne y leche debido a la reconversión de áreas de pastaje al cultivo de la soya, efecto que se ha hecho presente sobre todo en Argentina a escala nacional (Sades, 2008), país que de ser exportador de alimentos, especialmente de carne bovina y trigo, pasó a suspender estas exportaciones en marzo de 2007 por falta de producción nacional (Rulli y Boy, 2008). Segundo, la mayor parte de la soya cultivada es transgénica -la variedad conocida como soya Roundup Ready introducida a mediados de los años noventa, resistente al herbicida glifosfato, agente utilizado para eliminar otras plantas que no sean la soya en los campos de cultivo sin necesidad de tratamientos con maquinaria agrícola y que permite la siembra directa. Este paquete tecnológico tiene como características la necesidad de extensas áreas (la rentabilidad del cultivo aumenta con el aumento del área destinada a este) y de fumigaciones a gran escala con glifosfato o mezclas de herbicidas que lo contienen. La variedad transgénica alcanza hasta el 90\% de la producción total de soya, aunque en algunos países (por ejemplo, Paraguay) la totalidad de la soya sembrada es de este tipo. Estas características a su vez han provocado la desaparición masiva de pequeños agricultores, sobre todo en Argentina, por las dificultades que representa para las pequeñas empresas competir con la agricultura en gran escala, por lo cual los propietarios de pequeños lotes de tierra optan por alquilarlos a los pools soyeros.

Las intensas fumigaciones a que son sometidos los campos de soya son también un factor de gran presión socioambiental. Para empezar, la aplicación constante de agrotóxicos ha impactado severamente la fauna del suelo en que crece la soya pero, aparte de esto ya se han registrado casos de aparición de especies (principalmente plagas) resistentes a estos agentes, lo que exige aplicar más químicos por hectárea de cultivo. Otro aspecto importante de las fumigaciones, es que en algunos casos estas son realizadas en cercanías inmediatas de pueblos rurales, aumentando drásticamente la incidencia de enfermedades originadas por el contacto con sustancias tóxicas. Así, en Brasil un estudio (Pignati y Machado, 2007 apud REPORTER BRASIL, 2008) realizado en el estado de Mato Grosso, uno de los principales estados en que se cultiva la soya, in-

\footnotetext{
Especie de fondos de inversión en la agricultura, en los que se alquilan la tierra y la maquinaria para la realización de las labores agrícolas con el fin de generar un alto rendimiento a gran escala.
} 
dica que el aumento de la mortalidad por neoplasia y malformaciones congénitas pudiera estar relacionado con el aumento del uso de agroquímicos.

El avance de la soya es también responsable de la desaparición del empleo rural. En palabras de Rulli y Boy (2007) el modelo de la soya representa "una agricultura sin agricultores", ya que la soya, sobre todo en forma de paquete tecnológico, es uno de los cultivos que menos empleo generan: en Argentina la aplicación de tecnología hace necesario emplear tan solo a dos personas por hectárea al año (Rulli, 2007). Esto ha provocado una «descampesinización» extrema del campo argentino, obligando a los antiguos trabajadores rurales a migrar a los suburbios de las grandes ciudades argentinas en búsqueda de trabajo.

La expansión agresiva de la soya (alrededor de 700 mil nuevas hectáreas al año (Rulli y Boy, 2007) ampliamente incentivada por los gobiernos de los países sudamericanos se da no solo por la reconversión de áreas previamente destinadas a otros cultivos, sino también por una deforestación masiva. En Argentina, esta práctica en el período 1998-2002 alcanzó valores que fluctuaron entre $175 \mathrm{mil}$ y 200 mil hectáreas al año. En Brasil, el avance de la frontera de la soya es la principal causa de la desaparición paulatina del segundo bioma más importante -el Cerrado, del que actualmente queda un $33 \%$ de la superficie original (REPORTER, 2008), así como del Amazonas.
Estos efectos catastróficos del modelo de la soya han sido responsables del surgimiento de múltiples movimientos sociales, tanto contra el cultivo de la soya (de actores relacionados de una forma u otra con el campo) como contra las consecuencias socioeconómicas de la aplicación a escala nacional del modelo agroexportador -entre otras, el aumento del desempleo y de la pobreza, y la disminución de la población rural dedicada a la agricultura.

En la década de 1991 a 2001, cuando se aplicaron políticas neoliberales de desregularización del control estatal, dejando la economía nacional a merced del mercado internacional, estallan en Argentina numerosas protestas sociales que alcanzan una magnitud no vista anteriormente. Las protestas se dan en casi todo el territorio del país, ocupando un papel importante en el escenario nacional las generadas por los actores de origen agrario y residentes de poblados pequeños y ciudades de la provincia, que crecieron de la periferia al centro (Giarraca, 2002). Las protestas apuntaban a preservar los derechos sociales adquiridos durante el siglo anterior: condiciones de trabajo, salarios dignos, educación pública, derecho a conservar el patrimonio familiar, demanda de un ingreso mínimo frente a la pérdida del trabajo (en la lucha de los desocupados) y rara vez se relacionaron con la pretensión de nuevos derechos.

En este contexto aparecen actores que comienzan la lucha contra el 
modelo del cultivo de la soya -reflejo más fehaciente de la realidad neoliberal impuesta en Argentina. Ejemplo de estos actores es el Grupo de Reflexión Rural de Argentina (GRR, http://www. grr.org.ar), constituido a mediados de los años noventa "como un grupo de afinidad y un espacio de diálogos y debates multidisciplinarios sobre los impactos del capitalismo global» en la sociedad (GRR, sitio web). Paulatinamente, debido a la persistencia de la situación de «catástrofe social» (ibid.), este actor se va transformando «en un grupo activista y de construcción de pensamiento popular» y dinamizador de «redes y espacios de gestión». Esta estrategia de acción, basada en la concienciación de las personas comunes a través de la explicación, del uso de la lógica (y no apelando a las emociones) y la difusión accesible del conocimiento científico, es similar a la empleada por el movimiento social antirepresas (y otros movimientos sociales) en su fase actual. El fin perseguido es generar una visión alternativa a la oficial sobre la realidad vivida por la sociedad, que les permita a las personas identificar y elegir de manera consciente un modo de actuar ante las presiones creadas y ejercidas por el mercado. Se promueven valores nuevos, vinculados ya con los mecanismos de que depende la supervivencia del hombre en cualquier parte del mundo: la importancia de que la posibilidad de poder alimentarse no dependa de otro país o de los vaivenes del mercado, la importancia de tener acceso libre al agua potable, la importancia de poder decidir qué entorno legar a los hijos, la diversidad como base para el desarrollo humano.

El alcance de este marco interpretativo tan amplio es capaz ya de provocar resonancia en casi todos los sectores de la sociedad, en los distintos movimientos que reflejan las preocupaciones específicas de diversos actores sociales, sometidos a la misma presión bajo la cual se comienza a vislumbrar la necesidad de luchar por la supervivencia misma, de preservar lo suyo, la identidad, la cultura ante el avance homogeneizador del mercado neoliberal. Los valores proclamados llegan al punto de ser suficientes para unir bajo su poder de movilización a las sociedades de los países no solo de América Latina, sino del mundo.

Ya no se trata únicamente de eliminar el complejo soya y sus consecuencias, lo que demandan movimientos sociales como el Grupo de Reflexión Rural en Argentina. Su demanda principal ya es otra, expresada como slogan recientemente durante la Cumbre sobre Cambio Climático, celebrada en Copenhague en diciembre del 2009: «¡Cambien el sistema, no el clima!».

Se considera que el Estado y los gobiernos no son actores capaces de resolver esta demanda de cambio total de actitud y de valores. El Estado es tan solo una parte del sistema, la parte nacional, que se ha mostrado incapaz de desprenderse por si sola de los valores que trae el neoliberalismo: el dinero, el poder, el consumismo, la competencia. El proceso de cambio 
de valores se ha iniciado desde abajo, desde la sociedad latinoamericana, no buscando crear un poder alternativo para el Estado, sino una propuesta alternativa de sociedad con poder.

«El Planeta Tierra vive un período de intensas transformaciones técnicocientíficas como contrapartida de las cuales se han engendrado fenómenos de desequilibrio ecológico que amenazan, a corto plazo, si no se le pone remedio, la implantación de la vida sobre su superficie. Paralelamente a estas conmociones, los modos de vida humanos, individuales y colectivos, evolucionan en el sentido de un progresivo deterioro. Las redes de parentesco tienden a reducirse al mínimo, la vida doméstica está gangrenada por el consumo «massmediático», la vida conyugal y familiar se encuentra a menudo "cosificada» por una especie de estandarización de los comportamientos, las relaciones de vecindad quedan generalmente reducidas a su más pobre expresión... [...] Se trata una vez más como tantas veces en la historia, de que tendremos que luchar para sobrevivir ${ }^{7}$.

«El Estado mientras tanto, generalmente ausente, dejando hacer, cuando no, comprometido en políticas públicas tanto o más aterradoras, que aquellas que las mismas empresas podrían llevar adelante» $^{8}$.

7 Guattari, Félix (1996), «Las Tres Ecologías», Pre-Textos, Valencia, p.7. Editorial del domingo 3 de enero del 2010. Horizonte Sur, programa radiofónico del Grupo de Reflexión Rural, Argentina. http://horizontesurblog.blogspot. com/2010/01/editorial-del-domingo-3-deenero-de.html 13 de enero de 2010.

8 Editorial del domingo 3 de enero del 2010.
«En el contexto de esas preocupaciones, crece en el mundo el respeto por los agricultores familiares, las poblaciones campesinas subsistentes, los neorrurales que vuelven a la tierra desde las ciudades, así como por los pescadores artesanales o de costas, que son reconocidos en todos los foros internacionales como factor insoslayable, de la preservación de la biodiversidad [...].

La construcción de una modernidad tardía y de un socialcapitalismo sustentado en el respaldo de las Corporaciones, son objetivos que han comprometido a una generación de intelectuales de la izquierda progresista a todo lo largo del continente, y que introducen a nuestra América en un camino incierto y de difíciles retornos. De continuar ese camino, estamos sacrificando la originalidad de América, desconociendo la posibilidad de innovar y de imaginar un mundo nuevo, un mundo donde quepan muchos mundos diversos» ${ }^{9}$.

«A veces, los movimientos sociales, los movimientos revolucionarios $-\mathrm{O}$ como usted quiera llamarlos- se dividen porque se ponen a repartir un pastel que todavía no tienen.

La experiencia nos ha dicho que lo importante es crear poder popular desde abajo. Ahí está la clave de todo. Cambiar todas las cosas que están pasando no se logra poniendo un presidente del

Horizonte Sur, programa radiofónico del Grupo de Reflexión Rural, Argentina. http://horizontesurblog.blogspot. com/2010/01/editorial-del-domingo-3-deenero-de.html 13 de enero de 2010.

9 Editorial del domingo 27 de diciembre del 2009. Horizonte Sur, programa radiofónico del Grupo de Reflexión Rural, Argentina. http://horizontesurblog.blogspot. com/2009/12/editorial-del-domingo-27de-diciembre.html 13 de enero de 2010. 
Emma Mendoza eT aL. • Energías renovables y movimientos sociales...

grupo social al que usted pertenece. No es así como se van a resolver los problemas» ${ }^{10}$.

"Yo trabajo en una transnacional. Ahí se envasa Coca Cola, la cerveza, Pepsi Cola, los rones y el aguardiente. Un día me dice un compañero: 'Oíme vos, estas transnacionales ya no las aguantamos. ¿Vos por qué no buscás la manera de ser gerente, para que los problemas se nos acaben?' Lo mismo sucede en los movimientos sociales. Hay quienes dicen: 'Llevemos nuestro presidente para que se acaben los problemas'. No. Esto es un problema de estructura, y esta estructura que se ha construido en tanto tiempo sólo la vamos a destruir desde abajo» ${ }^{11}$.

¿Según su percepción política, solamente en América Latina es donde se están dando procesos de resistencia y de transformación. ¿El resto del mundo está rezagado?

«Es un hecho que las fuerzas de transformación en otras partes del mundo no han logrado aún una real posibilidad de realizar cambios. En Asia todavía el neoliberalismo es el modelo predominante en los denominados tigres asiáticos, aun en la China, Vietnam, o la India, el capitalismo es visto como oportunidad y no como agresión por

10 Entrevista a Carlos H. Reyes, líder del movimiento social hondureño contra el TLC, Coordinadora de Resistencia Popular. En: Grigsby W.(2004), «Nuevos movimientos sociales empiezan a renacer», envío digital. Nicaragua: Universidad Centroamericana, http://www.envio.org. ni/articulo/2189, 12 de enero de 2010.

11 la mayoría de la gente, porque se piensa que va a permitir un cierto desarrollo» ${ }^{12}$.

«La primera década del siglo XXI ha marcado ya una singular época de transformaciones en las Américas. El nuestro es un continente en ebullición, donde resistencias y revoluciones de nuevo tipo se anudan en la búsqueda de una sociedad nueva y al mismo tiempo arraigada en lo propio, en la construcción soberana de proyectos de vida en común que dejen atrás la explotación capitalista, el dominio imperial y colonial, la violencia patriarcal y racista, las guerras, la destrucción de pueblos y culturas, la depredación de la naturaleza» ${ }^{13}$.

\section{Conclusiones}

En el presente artículo examinamos brevemente el desarrollo de tres industrias de energía renovables que en la actualidad tienen su auge en la región y el impacto de este desarrollo en el

12 Arellano Ortiz F. «Para construir una nueva sociedad es imprescindible deslegitimar el capitalismo", Entrevista a Francois Houtart, profesor emérito de la Universidad Católica de Lovaina, director del Centro Tricontinental, secretario ejecutivo del Foro Mundial de Alternativas, miembro del Consejo Internacional del Forum Social Mundial de Porto Alegre, presidente de la Liga Internacional por el Derecho y la Libertad de los Pueblos y director de la Casa Editorial Ruth, Houtart. Cronicón. Sin fecha. http://www.cronicon.net/paginas/edicanter/ediciones23/nota1.htm

13 Declaración del Foro Social de las Américas-Consejo Hemisférico, 8 de Octubre de 2009. http://www.forosocialamericas.org 13 de enero de 2010. 
marco interpretativo que adoptan los movimientos sociales que emergen contra estas industrias. La hipótesis principal planteada consiste en que conforme aumenta la presión que ejerce cada una de estas industrias en la sociedad y el medio ambiente, aumenta también la oposición de los movimientos sociales, sufriendo sus demandas un cambio cualitativo. La lucha inicial por los derechos y la justicia es reemplazada paulatinamente por una lucha con objetivos más amplios, que tienen en su base la oposición al modelo económico extractivo instalado en América Latina con las reformas neoliberales de los años noventa. En este proceso el Estado juega un papel clave -por una parte, la democratización de los regímenes genera oportunidades políticas para la acción de estos movimientos sociales pero, por la otra, la actitud del Estado es uno más de los factores que exacerba la acción de estos movimientos que actúan contra las industrias de energía renovable. El vínculo del Estado con el sistema mismo que promueve el desarrollo de industrias extractivas lo torna pasivo ante la búsqueda de soluciones reales a los males que afligen a la sociedad, por lo que los movimientos sociales optan por una transformación de la sociedad desde adentro. Como vemos, los movimientos sociales evolucionan hacia una unión entre distintos tipos de actores que buscan en esta unión el logro de la supervivencia social. La sociedad se abre paso en el escenario internacional como actor para decidir cuáles serán los valores, las bases para su futuro desarrollo.

Para entender este proceso nuevo, de evolución de la sociedad desde adentro, es necesario incorporar el concepto de presiones como fuerzas ejercidas por las necesidades biológicas (internas, personales), morales (capaces de ser internalizadas por los sujetos) y sociales (externas, presiones sobre grupos sociales) determinadas por la naturaleza y por los diferentes actores sociales de distinto nivel en su interacción en el espacio de vida. La descripción detallada de este concepto queda fuera de los propósitos del presente artículo, pese a lo cual hay que resaltar una de las características de las presiones internalizadas: las presiones susceptibles de ser internalizadas dependen del conjunto de valores individuales del sujeto. Otra característica importante de las presiones es su jerarquía: las presiones internas o internalizadas, personales, son más fuertes, tienen mayor peso en la determinación de la conducta del sujeto que las presiones sociales externas. La adopción de valores nuevos para el individuo trae consigo la internalización y aceptación también de las presiones derivadas de esos valores. Por lo tanto, un movimiento social puede ser visto como un grupo de actores con valores internos similares que responden de manera unísona a una presión internalizada común. Por otra parte, se puede deducir que una presión que toque los valores más importantes, internalizados como básicos para el ser humano, 
tendrá mayor respuesta de parte de todas las personas que los comparten, de la sociedad en general. Estos valores, evidentemente, se relacionan con los mecanismos de que depende la supervivencia humana y, a nuestro juicio, son los que se están viendo afectados por el modelo neoliberal.

\section{BibLIOGRAFÍA}

[R]evolución Energética. Perspectiva Mundial de la Energía Renovable. Greenpeace Internacional, Consejo Europeo de Energía Renovables (EREC), 2007.

Arellano Ortiz, F. Para construir una nueva sociedad es imprescindible deslegitimar el capitalismo. Entrevista a Francois Houtart. Cronicón. Sin fecha. http://www. cronicon.net/paginas/edicanter/ediciones23/nota1.htm

Castañeda N. y L. Van Der Fleirt (2009),. Estudio sobre el impacto social y medioambiental de las inversiones europeas en México y Europa en el sector agua y electricidad. Iniciativa de Copenhague para Centroamérica y México (CIFCA), Red Mexicana de Acción Frente al Libre Comercio (RMALC). Marzo de 2007. Disponible en: http://www. rmalc.org.mx/documentos/FIANL..

Castro S.G. (2007) El Agua y los Ríos Amenazados en México. Los retos para el Movimiento Social Anti-Represas, Chiapas, MAPDER.

CMNUCC, Convención Marco de las Naciones Unidas sobre Cambio Climático. http:// unfccc.int, visitado en diciembre de 2009.

De Ita A. (2003) México: Impacto del PROCEDE en los conflictos agrarios y la concentración de la tierra. Centro de Estudios para el Cambio en el Campo Mexicano.

FORO SOCIAL DE LAS AMÉRICAS, http://www. forosocialamericas.org.

Goffman E., (1974), Frame Analysis: An Essay on the Organization of Experience. Nueva York, Harper.
Guattari F. (1996), Las Tres Ecologías, Valencia,. Pre-Textos..

Grisby W.,(2004) Nuevos movimientos sociales empiezan a renacer. Envío digital. Nicaragua: Universidad Centroamericana, http://www.envio.org.ni/articulo/2189

Habermas j. (1986), Ciencia y técnica como ideología. Madrid, Tecnos.

Honty G., Gudynas (2007), Agrocombustibles y Desarrollo Sostenible en América Latina y el Caribe. Situación, desafíos y opciones de acción. Observatorio del Desarrollo, Centro Latinoamericano de Ecología Social (CLAES). Disponible en http://www.ambiental.net/claes, visitado el 13 de diciembre de 2008.

HORIZONTE SUR, Programa radiofónico elaborado por el Grupo de Reflexión Rural, Argentina. Disponible en línea http:// horizontesurblog.blogspot.com

Informe de Estadísticas Energéticas 2006 (IEE) (2007), Quito,. OLADE.

LAWEA, Asociación Latinoamericana de energía eólica (2008). Disponible en línea: http://www.lawea.org, 20 de marzo de 2009.

Movimento dos Atingidos por Barragems (МAB),. Disponible en http://www.mabnacional.org.br/ 26 de marzo de 2009.

MAPDER (2008) Temacapul'n Declaration. Temacapul'n, Jalisco, México, 21 de junio.

McCormick S. (2004),. The Brazilian AntiDam Movement: Constructing a New Arena of Communicative Action. Ponencia presentada en el encuentro anual de la American Sociological Association, San Francisco, 14 de agosto.

Moreno Sada J.J. (2004) Corredor Eólico del Istmo, Comunidad de Bienes de Capital,. Gobierno del Estado de Oaxaca..

Nitsch, J.; W.Krewitt, M. Nast, P. Viebahn, S. Gärtner, M. Pehnt, G. Reinhardt, R.Schmidt, A. Uihlein, C.; Barthel, C.; M. Fischedick, F. Merten y K. Scheurlen (2004),. Ökologisch optimierter ausbau der nutzung erneuerbarer Energien in Deutschland. En: Bundesministerium für Umwelt, Naturschutz und Reaktorsicherheit. Umweltpolitik, Köllen Druck. 
Pignati W., y J.O. Machado (2007), O agronegócio e seus impactos na saúde dos trabalhadores e da população do Estado do MatoGrosso. En Pignati, W., Os riscos, agravos e vigilância em saúde no espaço de desenvolvimento do agronegócio no Mato Grosso.Tesis de Doctorado, Río de Janeiro, Fiocruz/ENSP.

PNUD (2007), Informe sobre Desarrollo Humano. México 2006-2007. Migración y desarrollo humano. .

Prefeitura Municipal de Osório (2008) «A natureza e a alta tecnologia em harmonia em um projeto que desperta atenção internacional.» Artículo en línea disponible en http://www.osorio.rs.gov.br/?static=eol/eolica2.htm, visitado el 20 de marzo de 2009.

Pronunciamiento del Foro Regional Parque Eólico del Istmo: Impactos ambiental, económico, social y cultural de los proyectos privados de energía eólica. Unión Hidalgo, Oaxaca, México. 25 de septiembre de 2005. Disponible en: http://www.ecoportal.net/content/view/full/53606, visitado el 21 de marzo de 2009

Red Latinoamericana contra Represas y por los Ríos, sus Comunidades y el Agua. (REDLAR). Disponible en: http://www.redlar. org, visitado el 26 de marzo de 2009.

Rein M. y D. Schön (1993), «Reframing policy discourse». En: The argumentative turn on policy analysis and planning. Fischer F. y J. Forester (eds.), Durham, NC, Duke University Press.

REPORTER BRASIL. (2008) El Brasil de los Agrocombustibles. Impactos de los cultivos sobre la tierra, el medio ambiente y la sociedad. Disponible en http://www. reporterbrasil.org.br, visitado el 18 de marzo de 2009
Rulli J. (2007), Introducción al Modelo de la Soya. En: Rulli J. (coord.) Repúblicas Unidas de la Soja. Realidades sobre la producción de soja en América del Sur. Grupo de Reflexión Rural, Argentina. Disponible en http://www.lasojamata.org, visitado el 1 de abril de 2009.

Rothman F. (1993), Political Process and Peasant Oppostion to Large Hydroelectric Dams: The case of the rio Uruguai movement in Southern Brazil, 1979 to 1992, Cambridge, Mass., Cambridge University Press.

Rothman F. D. y P. F. Oliver (1999) «From Local to Global: The Anti-Dam Movement in Southern Brazil, 1979-1992», Mobilization: An International Quarterly. V.4, N.1, pp. 41-57.

Rulli J. E. y A. Boy (2007), "Monocultivos y Monocultura: La pérdida de soberanía alimentaria». En: Rulli J. (coord.) Repúblicas Unidas de la Soja. Realidades sobre la producción de soja en América del Sur. Grupo de Reflexión Rural., Argentina. Disponible en http://www.lasojamata.org, visitado el 1 de abril de 2009.

Secretaría de Ambiente y Desarrollo Sustentable (SADES, Argentina) (2008), El Avance de la Frontera Agropecuaria y sus Consecuencias. Dirección Nacional de Ordenamiento Ambiental y Conservación de la Biodiversidad, Secretaría de Ambiente y Desarrollo Sustentable..

Snow D. y R, Benford (1988) «Ideology, frame resonance, and participant mobilization», International Social Movement Research. V.1, pp. 197-217. 